\title{
Hubble Space Telescope Time\#Series Photometry of the Transiting Planet of HD 209458
}

\section{Citation}

Brown, Timothy M., David Charbonneau, Ronald L. Gilliland, Robert W. Noyes, and Adam Burrows. 2001. "Hubble Space Telescope Time\#Series Photometry of the Transiting Planet of HD 209458." The Astrophysical Journal 552 (2): 699-709. https://doi.org/10.1086/320580.

\section{Permanent link}

http://nrs.harvard.edu/urn-3:HUL.InstRepos:41397423

\section{Terms of Use}

This article was downloaded from Harvard University's DASH repository, and is made available under the terms and conditions applicable to Other Posted Material, as set forth at http:// nrs.harvard.edu/urn-3:HUL.InstRepos:dash.current.terms-of-use\#LAA

\section{Share Your Story}

The Harvard community has made this article openly available.

Please share how this access benefits you. Submit a story.

Accessibility 
The Astrophysical Journal, 552:699-709, 2001 May 10

(C) 2001. The American Astronomical Society. All rights reserved. Printed in U.S.A.

\title{
HUBBLE SPACE TELESCOPE TIME-SERIES PHOTOMETRY OF THE TRANSITING PLANET OF HD $209458^{1}$
}

\author{
Timothy M. Brown, ${ }^{2}$ David Charbonneau, ${ }^{2,3}$ Ronald L. Gilliland, ${ }^{4}$ Robert W. Noyes, ${ }^{3}$ And Adam Burrows ${ }^{5}$ \\ Received 2000 November 21 ; accepted 2001 January 18
}

\begin{abstract}
We have observed four transits of the planet of HD 209458 using the STIS spectrograph on the Hubble Space Telescope (HST). Summing the recorded counts over wavelength between 582 and $638 \mathrm{~nm}$ yields a photometric time series with $80 \mathrm{~s}$ time sampling and relative precision of about $1.1 \times 10^{-4}$ per sample. The folded light curve can be fitted within observational errors using a model consisting of an opaque circular planet transiting a limb-darkened stellar disk. In this way we estimate the planetary radius $R_{p}=1.347 \pm 0.060 R_{\mathrm{Jup}}$, the orbital inclination $i=86^{\circ} .6 \pm 0.14$, the stellar radius $R_{*}=1.146$ $\pm 0.050 R_{\odot}$, and one parameter describing the stellar limb darkening. Our estimated radius is smaller than those from earlier studies but is consistent within measurement errors and also with theoretical estimates of the radii of irradiated Jupiter-like planets. Satellites or rings orbiting the planet would, if large enough, be apparent from distortions of the light curve or from irregularities in the transit timings. We find no evidence for either satellites or rings, with upper limits on satellite radius and mass of $1.2 R_{\oplus}$ and $3 M_{\oplus}$, respectively. Opaque rings, if present, must be smaller than 1.8 planetary radii in radial extent. The high level of photometric precision attained in this experiment confirms the feasibility of photometric detection of Earth-sized planets circling Sun-like stars.
\end{abstract}

Subject headings: binaries: eclipsing — planetary systems — stars: individual (HD 209458) techniques: photometric

\section{INTRODUCTION}

The low-mass companion to HD 209458 is the first extrasolar planet found to transit the disk of its parent star (Charbonneau et al. 2000; Henry et al. 2000). The primary star (G0 V, $V=7.64, B-V=0.58$; Høg et al. 2000) lies at distance of $47 \mathrm{pc}$ as determined by Hipparcos (Perryman et al. 1997). An analysis of radial velocity measurements by Mazeh et al. (2000) gave an orbital period of 3.524 days, with $M_{p} \sin i=0.69 \pm 0.05 M_{\mathrm{Jup}}$ and $a=0.0468 \mathrm{AU}$, using the derived value of $1.1 \pm 0.1 M_{\odot}$ for the stellar mass. When combined with the early photometric light-curve data, the same analysis yielded an orbital inclination $i=$ $86.1 \pm 1.6$ and a planetary radius $R_{p}=1.40 \pm 0.17 R_{\mathrm{Jup}}$. The planetary radius is at once the most interesting and the most uncertain of these parameters, largely because of uncertainty in the value of the stellar radius $R_{*}$. Knowledge of $R_{p}$ is important because it allows inferences about the planet's composition and evolutionary history (Guillot et al. 1996; Guillot 1999; Burrows et al. 2000). Unfortunately, the measured quantity that emerges most easily from the photometric transit data is the ratio $R_{p} / R_{*}$, and residual errors in the astrometry and effective stellar temperature

\footnotetext{
${ }^{1}$ Based on observations with the NASA/ESA Hubble Space Telescope, obtained at the Space Telescope Science Institute, which is operated by the Association of Universities for Research in Astronomy, Inc. under NASA contract NAS 5-26555.

${ }^{2}$ High Altitude Observatory/National Center for Atmospheric Research, 3450 Mitchell Lane, Boulder, CO 80307. The National Center for Atmospheric Research is sponsored by the National Science Foundation; timbrown@hao.ucar.edu.

${ }^{3}$ Harvard-Smithsonian Center for Astrophysics, 60 Garden Street, Cambridge, MA 02138; dcharbonneau@cfa.harvard.edu, rnoyes@ cfa.harvard.edu..

${ }^{4}$ Space Telescope Science Institute, 3700 San Martin Drive, Baltimore, MD 21218, gillil@stsci.edu..

${ }^{5}$ Department of Astronomy, University of Arizona, 933 North Cherry Avenue, Tucson, AZ 85721; aburrows@as.arizona.edu.
}

suffice to make the estimate of $R_{*}$, and hence $R_{p}$, uncertain by about $10 \%$. Additional small errors in $R_{p}$ result from uncertainties about the stellar limb darkening. Jha et al. (2000) used multicolor photometric data to reduce these uncertainties, obtaining $R_{p}=1.55 \pm 0.10 R_{\mathrm{Jup}}$. Finally, analyses of Hipparcos photometric data by Castellano et al. (2000) and Robichon \& Arenou (2000) have given refined estimates of the orbital period.

Here we report the results of very precise photometric measurements of transits of HD 209458b, obtained using the STIS spectrograph on the Hubble Space Telescope $(H S T)$. The motivations for the study were (1) to obtain sufficiently accurate photometry to reduce the ambiguity between estimates of the stellar radius, planetary radius, orbital inclination, and stellar limb darkening, (2) to search for evidence of planetary satellites or circumplanetary rings, and (3) to search the stellar spectrum observed in and out of transit for features imposed by transmission of starlight through the outer parts of the planet's atmosphere (Seager \& Sasselov 2000; Brown 2001).

We discuss the photometric results [items (1) and (2) above] in the present work; the spectroscopic investigation [item (3) above] will be the subject of a later paper.

\section{OBSERVATIONS AND DATA ANALYSIS}

During a transit of HD 209458b, the apparent brightness of the star is reduced by a little less than $2 \%$ as a result of the light blocked by the gas-giant planet. To have a useful sensitivity to smaller objects such as circumplanetary rings or Earth-sized satellites, we required photometric accuracies at least 2 orders of magnitude better, with time resolution of a few minutes or less. Most instruments on $H S T$ cannot meet these requirements, because they are not designed to accept the requisite large photon fluxes. The Space Telescope Imaging Spectrograph (STIS), used in spectrographic mode with the CCD detector, is the exception. By dispersing the light in a large bandwidth over many 
pixels, using the $4 e^{-}$per data number gain setting, STIS can collect $2.5 \times 10^{8}$ photons per detector readout without saturating any detector pixels. A summation over all sampled wavelengths then provides a photometric signal whose shot-noise-limited signal-to-noise ratio (SNR) is above $10^{4}$. The readout time for a detector subarray of the necessary size $(1024 \times 64$ pixels $)$ is about $20 \mathrm{~s}$; it is thus possible to maintain both an $80 \mathrm{~s}$ sampling cadence and a respectable duty cycle of $75 \%$. To attain near shot-noise limited operation, one must control, or verify the smallness of, numerous sources of instrumental noise, such as variations in the shutter opening and closing times, and CCD gain variations combined with spectrum motion on the detector. One of us had already investigated the use of the STIS as a precise photometer, and had demonstrated that, with care, these systematic errors can be suppressed (Gilliland 1999; Gilliland, Goudfrooij, \& Kimble 1999). We note that these studies further demonstrated that time series with SNR $=10^{4}$ could be maintained even well past saturation of individual pixels, although in the present observations we did not saturate the detector.

We observed HD 209458 during each of four planetary transits, on UT dates 2000 April 25, April 28-29, May 5-6, and May 12-13. The full duration of a transit is 184.25 minutes, or slightly less than two HST orbits, which are 96.5 minutes each. Objects orbiting the planet may, however, precede or follow it by as much as $R_{H}$, the radius of the planet's Hill sphere (i.e., the radius at which the star's tidal forces would dislodge a satellite):

$$
R_{\mathrm{H}}=a\left(\frac{M_{p}}{3 M_{*}}\right)^{1 / 3}=4.2 \times 10^{5} \mathrm{~km}=5.9 R_{\mathrm{Jup}},
$$

where $a$ denotes the planet's orbital radius and $M_{p}$ and $M_{*}$ the masses of the planet and star. Since the planet's orbital speed is estimated to be $143 \mathrm{~km} \mathrm{~s}^{-1}$, one must search for gravitationally bound objects as much as 49 minutes, or about one-half of an HST orbit, before and after the transit central time. This requires four full HST orbits to assure adequate coverage before and after the planetary transit. To allow the telescope pointing and thermal environment to stabilize before beginning critical observations, we added one further orbit at the beginning of each transit sequence, for a total of five $H S T$ orbits for each transit. The orbital phasing was such that each observed transit contained two initial orbits that were completely off-transit, two orbits that fell almost entirely during the transit, and one following orbit that was off-transit.

We took all observations using the G750M grating, covering the wavelength range $581.3 \leq \lambda \leq 638.2 \mathrm{~nm}$, with a resolution of $R=\lambda / \Delta \lambda=5540$, corresponding to a resolution element of 2.0 pixels or $0.11 \mathrm{~nm}$. This wavelength range was chosen to cover the region of the $\mathrm{Na} \mathrm{D}$ lines, where a large signature from transmission through the planetary atmosphere is thought to be likely. We used a $52^{\prime \prime} \times 2^{\prime \prime}$ slit, much larger than the (typically 0".07 FWHM) stellar image size, to minimize variations in the fraction of stellar light lost at the slit edges due to minor guiding and focus changes. (We did not use the "clear" slit in order to avoid excessive sky background.) The CCD area read out consisted of a $1024 \times 64$ subarray, covering the entire possible range in the dispersion direction and about 3"2 across the dispersion. Exposure times were $60 \mathrm{~s}$, which with a $20 \mathrm{~s}$ readout time gave an observing cadence of 1 sample per 80 s. This exposure time gave about $1.55 \times 10^{8}$ detected photons per spectrum, which would correspond to an optimal photon-noise-limited precision of $8.0 \times 10^{-5}$, or 87 $\mu$ mag. Figure 1 shows a typical extracted stellar spectrum (see the discussion below for details of the extraction process). The first orbit of each group of five has a reduced number of spectra owing to time spent on target acquisition. During each visit of five orbits, we obtained 28 spectra during the first visit, then 36 spectra during each of the subsequent three orbits, then 35 spectra during the final orbit, for a total of 171 spectra. The final data set thus contained $4 \times 171=684$ individual spectra of HD 209458 . We took wavelength calibration spectra just before each of the five orbits, and, as an associated calibration program, we obtained 49 flat-field exposures during occultation time within the orbits surrounding the transit observations. All data for both the science program (8789) and calibration program (8797) are publicly available via the $H S T$ archive; ${ }^{6}$ there is no proprietary period for these data since they were obtained through Director's Discretionary time.

Observations of the first transit (UT 2000 April 25) were partly compromised by a database error in the location of the subarray for this rarely used secondary central wavelength setting of G750M. The result was that the spectrum was not entirely contained within the CCD subarray for the

${ }^{6}$ See http://archive.stsci.edu.

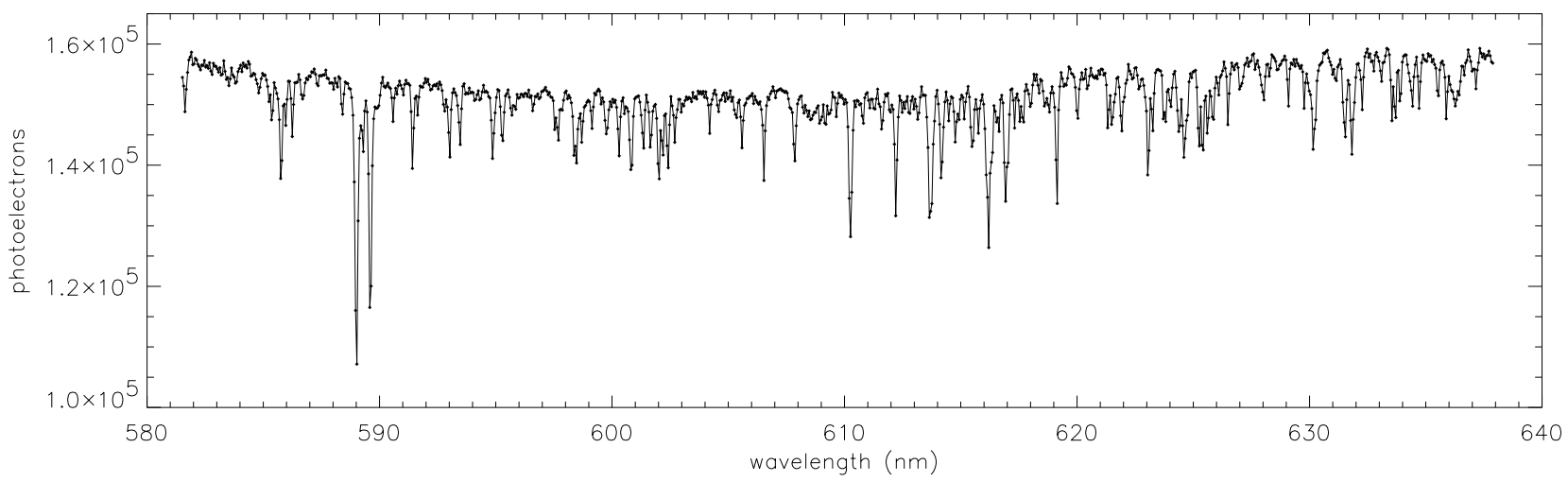

FIG. 1.-Typical spectrum of HD 209458 obtained with the STIS instrument, showing the number of detected photons in a single $60 \mathrm{~s}$ integration vs. wavelength. We obtained 684 such spectra in all. 
red half of the spectral range. This error led to reduced counts and increased sensitivity to cross-dispersion positional drifts over this range. In the subsequent data analysis, we ignored this part of the wavelength range, but only for the first transit. Rapid work by the staff at STScI identified the source of this error and provided an effective correction strategy (a real-time slew executed after target acquisition for the visit on April 28-29, and a correction to the database thereafter), so that the spectra for subsequent transits were correctly positioned on the subarray.

Data reduction consisted of (1) recalibrating the twodimensional CCD images, (2) removing cosmic-ray events, (3) extracting one-dimensional spectra, (4) summing the detected counts over wavelength to yield a photometric index, and (5) correcting the resulting photometric time series for variations that depend on the phase of the HST orbit and for variations between visits.

Recalibration of the CCD images started from the biasand dark-subtracted and flat-fielded images produced by the standard STIS data reduction pipeline. The STIS pipeline builds a weekly bias image from exposures taken for this purpose. We examined the biases that were subtracted from each image and found them to be sufficiently accurate. In contrast to this, the pixel-to-pixel flat field images are often produced from data that are several months old, and we found that the relative sensitivity of numerous pixels had changed since the most recent pipeline flats were produced. Furthermore, the pipeline flats did not have a sufficiently high SNR for our purposes. We requested that high SNR flats be taken during times of Earth-occultation contemporaneous with our science exposures. We shot two sets of seven such exposures for each of the visits on UT 2000 April 24, 27, and May 12, and one set of seven such exposures for the visit on UT 2000 May 5. For each set of seven exposures, the intensity in each exposure increased with time and in a reproducible manner from one set to the next. The origin of this increase is unknown, although a possibility is that the calibration lamp warmed slowly as the series of exposures progressed. This effect was removed by renormalizing by the total number of counts. The relative illumination among pixels by the continuum source was remarkably constant: No temporal drift was observed (aside from the normalization variation described above); thus we were able to quantify the relative sensitivity of individual pixels (which typically varied with an amplitude of $1 \%$ ) to a precision of better than $0.1 \%$. Between each visit, a small fraction of the pixels changed their relative sensitivity significantly, most likely owing to radiation events between visits. Furthermore, the STIS CCD was annealed on UT 2000 May 6, between the third and fourth visits, which changed the behavior of a number of pixels. To handle sensitivity variations between any two visits, we tagged all pixels whose sensitivity we observed (from the contemporaneous flats) to have changed and produced new flat fields for each visit where these pixels were replaced by their value derived from the data obtained during only the one visit. We then multiplied all the science exposures by the pipeline flat field, to undo the calibration from the pipeline, and then divided all the exposures by our newly derived flat fields. The calibration changes we imposed were typically small, typically a fraction of a percent in the gain of individual pixels. Since the photometric signal (described below) is produced by summing over a large number of pixels, these changes made only a small, but noticeable, improvement in the final results. In contrast to this, the improved precision from this recalibration provided significant gains in the analysis of the spectra to search for transmission features (to be presented in a separate paper).

Cosmic-ray hits on the CCD were a significant source of noise in the recalibrated data. We corrected for these by forming a time series of the intensity at each pixel and fitting this series as a fourth-order polynomial in time. Points showing statistically significant positive differences from the fit were deemed to be corrupted. Such points were replaced by the value of the fitted function at the corresponding time. Of the 65536 pixels in each image, typically 36 (this is the median number) had to be corrected in this way, and of these pixels about $25 \%$ contributed to the final photometric index for the image.

We extracted one-dimensional spectra simply by summing along CCD columns, taking at each column a band of fixed width centered on the measured crossdispersion spectrum position. Since motion of the spectrum in the cross-dispersion direction was measured to be much less than one pixel (typically motions were roughly 0.05 pixels within visits) for the entire data set, we used the same position for the integration band to produce all extracted spectra. We used a total cross-dispersion band 17 pixels in width, which we found produced the minimum rms variation in the photometric time series for orbits that occurred out of transit. The result of this operation was a onedimensional spectrum sampled at 1024 points.

For the most part we describe here a single photometric index, namely, a sum $S(t)$ of the one-dimensional spectrum over almost the entire available spectral range (581.9 to $614.6 \mathrm{~nm}$ for the first transit, and 581.9 to $637.6 \mathrm{~nm}$ for the others). The only refinement in this process was to position the endpoints of the summation so as to avoid obvious absorption lines, so that the sum would be insensitive to displacements along the dispersion. In addition we performed an experiment in which we summed separately over the red and blue halves of the wavelength range; the difference between these two light curves results from the color dependence of the stellar limb darkening. The details of this analysis are described in $\S 3.3$.

The time series $S(t)$ shows small but repeatable variations in phase with the $H S T$ orbital period. We do not know the origin of these variations, though we suspect that they are connected with the telescope's orbital thermal cycle. The variations are fairly well approximated by a linear decrease with an amplitude of $0.1 \%$ over 48 minutes, with some curvature at the beginning and end of each orbit. We corrected the time series on a transit-by-transit basis. For each transit, we phased the data from the out-of-transit orbits (orbits 2 and 5) to the $H S T$ orbital period. We then fitted a fourth-order polynomial to these data and divided all five orbits of the transit by this function. The first sample in every orbit is always smaller than the average by $0.25 \%$; we rejected these values from the time series.

The corrected time series have transit-to-transit differences of scale that are large for the first transit (because we summed over a smaller range of wavelength) and smaller (typically $0.1 \%$ ) for the remaining three transits. Lacking a comparison star or other external calibration source, we cannot say how much of this long-term variation arises from the star and how much from instrumental drift. For our purposes this is not important, however, since we are primarily concerned with variations on timescales of 


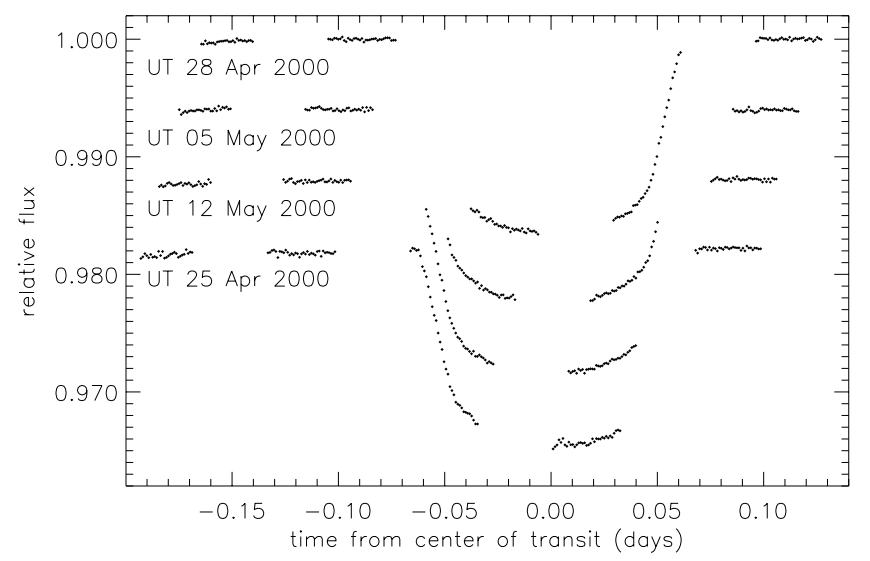

FIG. 2.-Time series of the corrected intensity shown separately for each of the four transits observed by HST, with successive transits offset by -0.006 for clarity. Note that, because the transit duration is almost two $H S T$ orbits, complete temporal coverage was not obtained for any one transit.

minutes or hours, and not weeks. These scale variations were also removed by the procedure described in the previous paragraph.

The normalized time series have a value of unity when averaged over the out-of-transit orbits, and minimum values near the transit centers of 0.9836 . Figure 2 shows the time series for each of the four transits phased with respect to the planetary orbit, and Figure 3 shows the combined data for all four phased transits, excluding the first orbit of each visit. One may estimate the noise in Figures 2 and 3 from the scatter among the out-of-transit observations. For the last three transits, these are typically $1.1 \times 10^{-4}$, or 120 $\mu$ mag for each $60 \mathrm{~s}$ integration; the noise for the first transit is worse by a factor of about 2.3, mostly because of increased sensitivity to transverse motions of the poorly centered spectra. This precision suffices to show the $1.64 \%$ transit dip with a SNR of about 150, at high time resolution.

\section{INTERPRETATION}

\subsection{Planetary Orbital Parameters}

Ideally, we would like to measure $T_{c}$, the time at the center of the transit, for each of the four observed transit events. However, we have gaps in our observations of any one transit (see Fig. 2) due to Earth occultation that prevent

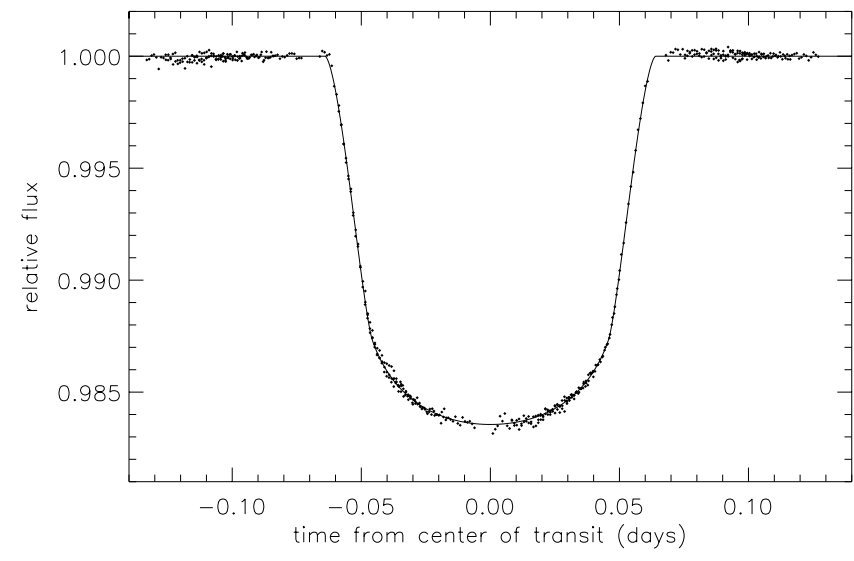

FIG. 3.-Phased light curve for all four transits, assuming a planetary orbital period of 3.52474 days. The time series for each transit has been scaled to have the same average intensity over the second and fifth (out-oftransit) orbits. us from doing so with sufficient accuracy. We can, however, estimate the period accurately (and independently from the absolute times of the transits) by seeing which assumed value of the period produces the minimum scatter in the phased transit curve. In this manner we derive an orbital period of $P=3.52474 \pm 0.00007$ days. Having evaluated the period, we can then subtract the appropriate multiple of it from each of visits 2,3 , and 4 (1,3, and 5 planetary orbital periods later, respectively), to phase the data to the time of the first transit. We then fold the transit curve about an assumed time $T_{c}$ for the midpoint, and derive $T_{c}$ from minimizing the scatter between the observation before and after the center of transit. We derive $T_{c}=2451659.93675$ $\pm 0.0001 \mathrm{HJD}$. We can then compare this value of $T_{c}$ with the three other accurately measured transit times, two from Charbonneau et al. (2000) and one from Jha et al. (2000). Doing so, we derive a more accurate value of the period, owing to the large number of transits that have now elapsed from these observation from fall 1999. We find $P=3.52480 \pm 0.00004$ days. The uncertainty in the period is $4.3 \mathrm{~s}$. Our derived value is in excellent agreement with the values derived with similar precision from Hipparcos archive photometry by Castellano et al. (2000), who found $P=3.524736 \pm 0.000045$ days, and by Robichon \& Arenou (2000), who found $P=3.524739 \pm 0.000014$ days.

\subsection{Stellar and Planetary Parameters}

To estimate the planet's radius, we sought to represent the light curve as the result of an opaque sphere of radius $R_{p}$ in an inclined circular orbit about a limb-darkened star of radius $R_{*}$. We took the stellar mass to be $1.1 \pm 0.1 M_{\odot}$ (Mazeh et al. 2000), with the orbital radius and velocity determined from the period and Kepler's laws. Free parameters in the fit were $R_{p}, R_{*}$, the orbital inclination $i$, and the parameters $u_{1}$ and $u_{2}$ describing the stellar limb darkening in the form

$$
\frac{I(\mu)}{I(1)}=1-u_{1}(1-\mu)-u_{2}(1-\mu)^{2},
$$

where $\mu$ is the cosine of the angle between the line of sight and the normal to the local stellar surface (Claret \& Gimenez 1990). A simpler and often-used parameterization replaces the right-hand side of equation (2) with $1-u(1-\mu)$. We find that this parameterization worsens the $\chi^{2}$ statistic of the fit slightly, but significantly. We note, however, that fitting the expression in equation (2) gives strongly anticorrelated formal errors for $u_{1}$ and $u_{2}$. Thus, a more natural set of limb-darkening parameters is $\left(u_{1}+u_{2}\right)$ and $\left(u_{1}-u_{2}\right)$. The former describes the total magnitude of the limb-darkening variation and is well constrained by the observations, while the latter describes the amount of curvature in the limb-darkening function and is relatively poorly constrained. For explanatory purposes below, we shall refer to these combinations rather than to $u_{1}$ and $u_{2}$ individually, and moreover we shall adopt the approximation that $u \simeq u_{1}+u_{2}$.

The conceptual basis for this fitting process is illustrated in Figure 4. At the lowest level of approximation, the light curve is described by just 2 parameters: its depth $d$ depends mostly upon $R_{p} / R_{*}$, while its duration $l$ depends mostly upon the transit's chord length, and therefore upon $R_{*}$ and $i$. At this treatment level there are fewer observables $\{d, l\}$ than unknowns $\left\{R_{p}, R_{*}, i\right\}$, and so one must estimate $R_{*}$ from other evidence in order to obtain values for $R_{p}$ and $i$. 


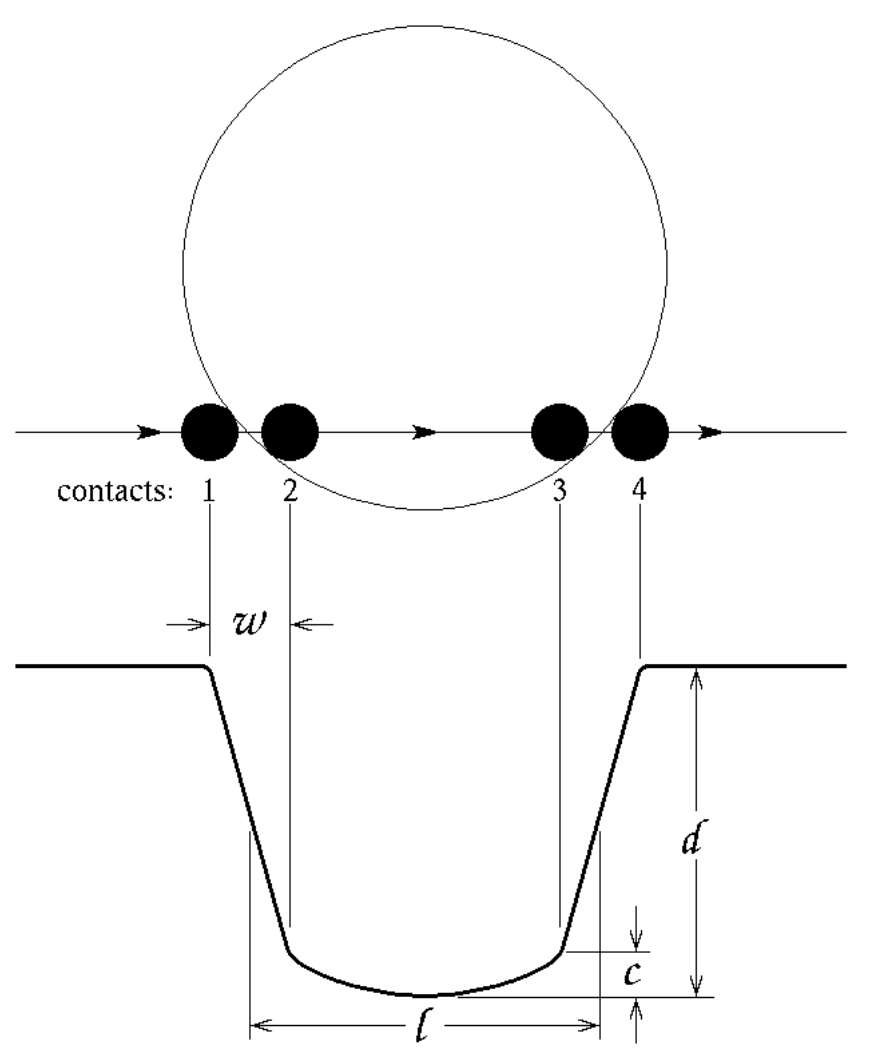

FIG. 4.-Schematic illustration of the light curve of a transiting planet. Measurable quantities are the duration of the transit $l$, the transit depth $d$, the ingress/egress duration $w$, and the central curvature of the light curve $C$. Given the orbital speed (which follows from the orbital period and the stellar mass), these quantities determine the radii of the star and of the planet, the orbital inclination, and the degree of limb darkening.

This fitting degeneracy can be removed by taking account of more subtle effects: The duration $w$ of the planet's ingress and egress depends upon $R_{p}$ but is also proportional to $\sec \psi$, where $\psi$ is the angle between the planet's line of motion and the local normal to the stellar limb. Thus, $w$ depends upon $R_{p}, R_{*}$, and $i$. Finally, the curvature $C$ of the light curve between second and third contacts depends upon the stellar limb-darkening parameter $u$ and upon $i$ and $R_{*}$. Thus, if $d, l, w$, and $C$ can be measured with adequate precision, one may estimate each of the four independent system parameters $R_{*}, R_{p}, i$, and $u$. Fitting for both $u_{1}$ and $u_{2}$ requires, in addition to the quantities already mentioned, a measurement of the detailed shape of the light curve between second and third contacts. All of the foregoing assumes that the star's mass $M_{*}$, and hence the planet's orbital velocity and semimajor axis, are known. The derived value of $R_{p}$ is only weakly dependent upon the assumed value of $M_{*}$, scaling as $R_{p} \propto M_{*}^{1 / 3}$. We assume $M_{*}=1.1 \pm 0.1 M_{\odot}$ (Mazeh et al. 2000), and our derived errors include this uncertainty in the stellar mass.

We derive best-fit values for $\left\{R_{p}, R_{*}, i, u_{1}, u_{2}\right\}$ by minimizing the $\chi^{2}$ of the fit. The reduced $\chi^{2}$ for the best-fit values was 1.07 , indicating that the model is a good fit to these data. To derive $1 \sigma$ errors for each parameter, we change the value of that parameter and fix it at a new value, and then allow all other parameters to float, as well as allow for a stellar mass between 1.0 and $1.2 M_{\odot}$. We repeat this procedure until the best-fit solution produces an increase in the $\chi^{2}$ corresponding to a $1 \sigma$ change. The best-fit parameters and their errors are given in Table 1. Both the stellar and
TABLE 1

HD 209458B Fit PARAMETERS

\begin{tabular}{cc}
\hline \hline Parameter & Value \\
\hline$R_{*} \ldots \ldots \ldots \ldots$. & $1.146 \pm 0.050 R_{\odot}$ \\
$R_{p} \ldots \ldots \ldots \ldots$. & $1.347 \pm 0.060 R_{\text {Jup }}$ \\
$i \ldots \ldots \ldots \ldots \ldots$ & $86.68 \pm 0.14$ \\
$\left(u_{1}+u_{2}\right) \ldots \ldots$ & $0.640 \pm 0.030$ \\
$\left(u_{1}-u_{2}\right) \ldots \ldots$ & $-0.055 \pm 0.100$ \\
\hline
\end{tabular}

planetary radii are found to be in agreement with those derived by Mazeh et al. (2000), and the precision has been increased greatly. Most significantly, the error in the planetary radius has been reduced from $0.17 R_{\mathrm{Jup}}$ to $0.06 R_{\mathrm{Jup}}$. We note also that our derived value for $R_{*}$ is in agreement with that derived from the Hipparcos distance, $R_{*}=1.18 \pm 0.09$ $R_{\odot}$ (see Jha et al. 2000 for details). The values for $R_{p}$ and $R_{*}$ derived here are somewhat smaller than those derived by analysis of multicolor data by Jha et al. (2000), although the differences are consistent with the errors, differing by less than $1.4 \sigma$. There are some correlations among the formal errors for the various derived parameters, and these are illustrated in Figure 5. We reiterate that our stated $1 \sigma$ errors include, and are dominated by, these correlations. In particular, the uncertainties in the radii of the planet and star are dominated by the assumed uncertainty of \pm 0.1 $M_{\odot}$ in the stellar mass.

\subsection{Color Dependence of the Light Curve}

To check for color dependence of the transit curve, we divided the spectral region in two ranges, 581.9-609.7 nm ("blue"), and 609.7-637.6 nm ("red"). We then generated photometric time series for each of these following the procedure described in $\S 2$. We used data from only visits 2,3 , and 4 , since the data from visit 1 lacked the red half. For each color, we phased the data to the period of 3.52474 days and folded it about the midpoint of the transit, $T_{c}$. Since we do not have useful data from the first visit, gaps exist in the time coverage. We then grouped the data into 5 minute bins, and for the data in each bin, we fitted brightness as a linear function of time within the bin. We took the brightness for the bin to be this linear function, evaluated at the central time for the bin. We then differenced the red and blue transit curves generated in this manner. These data are shown in Figure 6. From these data, it is clear that the transit is deeper in the blue at times near the center of transit, and deeper in the red at times when the planet is near the limb, as would be expected from the greater limbdarkening in the blue (see, e.g., Rosenblatt 1971 and Sackett 1999 for sample differenced-color light curves).

To generate the best-fit light curve for the difference of these color data, we fixed $R_{p}, R_{*}$, and $i$ at the values derived in $\S 3.1$. We then allowed small changes to the parameters describing the limb darkening, such that for the red half (denoted by $R$ ),

$$
\begin{aligned}
& \left(u_{1}+u_{2}\right)_{R}=\left(u_{1}+u_{2}\right)+\alpha, \\
& \left(u_{1}-u_{2}\right)_{R}=\left(u_{1}-u_{2}\right)+\beta,
\end{aligned}
$$

and for the blue half (denoted by $B$ ),

$$
\begin{aligned}
& \left(u_{1}+u_{2}\right)_{B}=\left(u_{1}+u_{2}\right)-\alpha, \\
& \left(u_{1}-u_{2}\right)_{B}=\left(u_{1}-u_{2}\right)-\beta .
\end{aligned}
$$



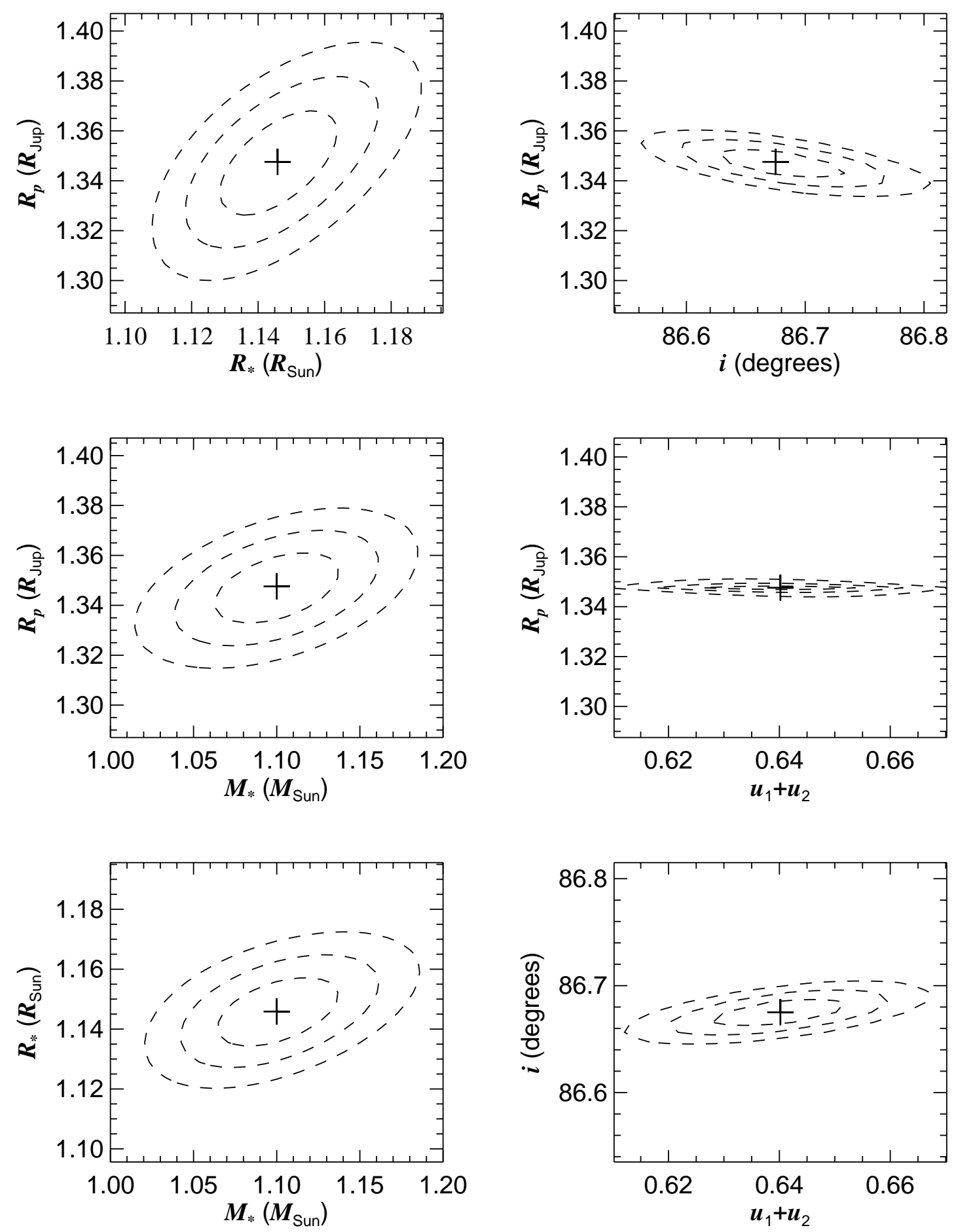

FIG. 5.-The $\chi^{2}$ surfaces for the fit of $R_{p}, R_{*}, i$, and $\left(u_{1}+u_{2}\right)$ to the observations. Each panel shows one two-dimensional cut through the five-dimensional $\chi^{2}$ surface, as indicated in the figure. The displayed ranges in each plot are the $1 \sigma$ error intervals in each case. The plotted contours serve to indicate the mutual dependences of the selected parameters, but the contour levels do not correspond to specific significance levels. 


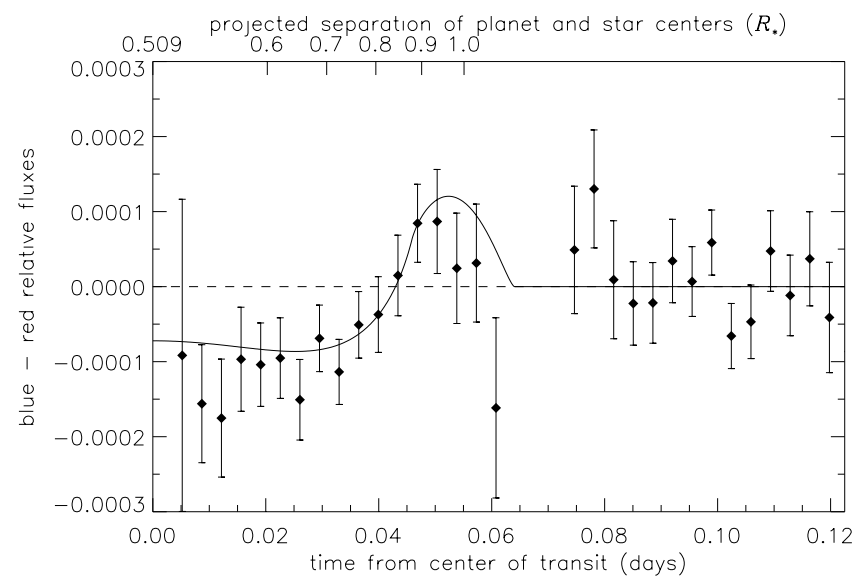

FIG. 6.-Color dependence of the transit light curve, shown as the difference (blue-red) between the normalized fluxes in the wavelength ranges $(581.9 \mathrm{~nm}, 609.7 \mathrm{~nm})$ ( blue) and $(609.7 \mathrm{~nm}, 637.6 \mathrm{~nm})($ red), plotted against time from transit center. The solid line corresponds to the best-fit model color-dependent limb-darkening law, as described by eq. (5).

We then generated model light curves for both the red and blue data, differenced these, and evaluated the $\chi^{2}$ of this fit to the data. The data are best fitted by $\alpha=-0.021$ and $\beta=0.063$; thus the derived values for the limb-darkening are

$$
\begin{gathered}
\left(u_{1}+u_{2}\right)_{R}=0.619 \pm 0.03 \\
\left(u_{1}+u_{2}\right)_{B}=0.661 \pm 0.03 \\
\left(u_{1}-u_{2}\right)_{R}=0.003 \pm 0.1, \\
\left(u_{1}-u_{2}\right)_{B}=-0.123 \pm 0.1,
\end{gathered}
$$

consistent with the measured solar limb-darkening (Cox 1999). We show this best-fit curve in Figure 6. Assuming the best-fit values for $i$ and $R_{*}$, we can calculate the projected separation of the center of the planet and the center of the star at any time during the transit, and thus at any point in this color curve. This axis is shown at the top of Figure 6 . From this one can determine the fractional stellar radius at which the limb-darkening curves (weighted over the stellar disk) cross; this occurs near a radius of $0.84 R_{*}$.

The color-dependent amplitude shown in Figure 6 is small primarily because we are contrasting the limbdarkening over two bands that are separated by only $28 \mathrm{~nm}$. Observing the transit in very disparate bands across the visible and near-IR would show a much greater effect. If one were willing to assume a model for the limb-darkening of the star, one could use this effect to break the degeneracy between the parameters $R_{p}, R_{*}$, and $i$, even if the data did not have the precision of that which we present here. This is precisely what was done with multicolor observations of HD 209458 in Jha et al. (2000).

\subsection{Search for Circumplanetary Rings}

If the planet of HD 209458 were circled by a ring system with significant opacity, the rings would cause distortions of the light curve relative to that of a spherical body (Schneider 1999). The cross-sectional area of the planet would appear larger because of the light obstructed by the rings, and (more usefully, for detection purposes) one would also see small dips in the light curve before first and after fourth contact (see Fig. 7). The phased light curve is fitted within observational errors by the simple planetary-transit model described above, so there is no evidence for rings in the current data. We can, however, set an upper limit on the size of a ring system consistent with the observations. For this purpose we assume that such a ring system lies in the planet's orbital plane, that it extends continuously from the planet's limb to a maximum radius $R_{r}$ (measured in units of $R_{p}$ ), and that it is entirely opaque to transmitted light. We cannot simply assume the best-fit values for $\left\{R_{p}, R_{*}, i, u_{1}, u_{2}\right\}$ from $\S 3.1$, because, if circumplanetary rings are present, some area is occulted by the rings; this would cause us to over-estimate $R_{p}$. To simplify the investigation, we fix $R_{*}, u_{1}$, and $u_{2}$. Changing the value of $R_{*}$ does not affect the results since it would result in a larger value of $R_{p}$, and we state an upper limit for $R_{r}$ in units of $R_{p}$. For each trial value of $R_{r}$, we allow $R_{p}$ and $i$ to float and derive an upper limit for $R_{r}$ by finding the value above which the $\chi^{2}$ increases by an amount corresponding to a $3 \sigma$ change. The maximum ring radius consistent with the observations at this confidence level is then $1.8 R_{p}$; this is slightly smaller than the radius of Saturn's ring system, measured in units of Saturn's radius. This fairly low sensitivity to ring systems results mostly from the assumption that rings must lie in the planet's orbital plane, and hence that the ring plane must be nearly edge-on as seen from Earth.

\subsection{Search for Planetary Satellites}

A satellite orbiting HD 209458b might be detectable either from its photometric signature or from its influence on the orbital motion of the planet itself (Sartoretti \& Schneider 1999). A satellite would block light in addition to that obstructed by the planet, unless it happened to be projected onto the planet's disk during the transit. This additional obstruction could occur either earlier or later than the main transit, depending upon the satellite's position in its orbit. Similarly, its duration could be slightly different from that of the planetary transit, because the satellite can move significantly in its orbit during the transit and because the chord the satellite strikes across the star may be longer or shorter than that of the planet.

As was the case for rings, there is no evidence for satellites in the photometric time series. Figure 8 shows the residuals about the best-fit light curve for each of the observed transits, along with transit curves that might be expected from a satellite with 1.5 times the radius of the Earth and an orbital period of 1.5 days. A signal of this size would be easily detected, if it were present. To set a better limit on the size of possible satellites, we searched the residual data for repeated transit-like events by applying matched filters that simulated the light curves from satellites with a range of orbital periods, phases, and semimajor axes. This process was analogous to that used to search for transiting planets in observations of the eclipsing binary CM Dra (Doyle et al. 2000), and in HST time-series photometry of the globular cluster 47 Tucanae (Gilliland et al. 2000; Brown et al. 2001). The process we actually used worked as follows: We first subtracted the best-fit model for the transit curve of the planet from the data, yielding the photometric residuals $R(i)$, with $i=[1, \ldots, N]$. We used the data only from the last three visits, as the errors due to the offset of the spectrum in the CCD subarray during the first transit are relatively very large. We constructed a dense grid of sample satellite orbital periods $(p \in[1 d, 3.5 d])$ and satellite orbital phases $(\phi \in[0$, $1])$. For each pair of $\{p, \phi\}$, we evaluated, for each visit, the 

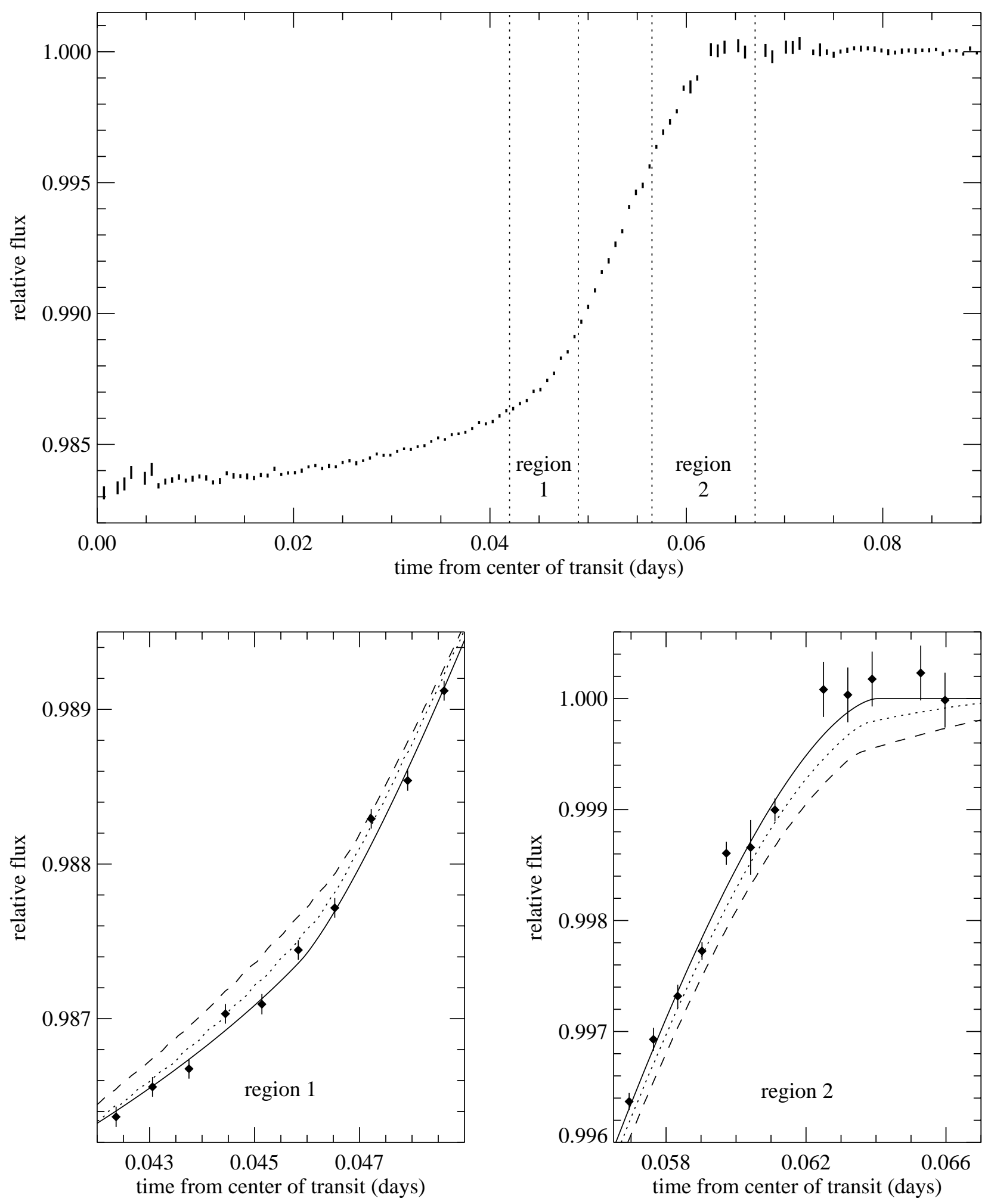

FIG. 7.- - Upper panel: Observed light curve folded around the central transit time (bars) binned to $60 \mathrm{~s}$ sampling. The bar height indicates the formal errors of the binned estimates. Regions 1 and 2 are shown at larger scale in the lower panels. Lower panels: Observed intensities (filled diamonds with $1 \sigma$ error bars) and curves for best-fit models assuming maximum ring radii of $1 R_{p}$ (i.e., no rings) (solid curve), $1.8 R_{p}$ (dotted curve), and $2.2 R_{p}$ (dashed curve). The latter two curves are inconsistent with the data. Rings were assumed to be opaque, to lie in the planet's orbital plane, and to extend from $1 R_{p}$ to the stated radii. 


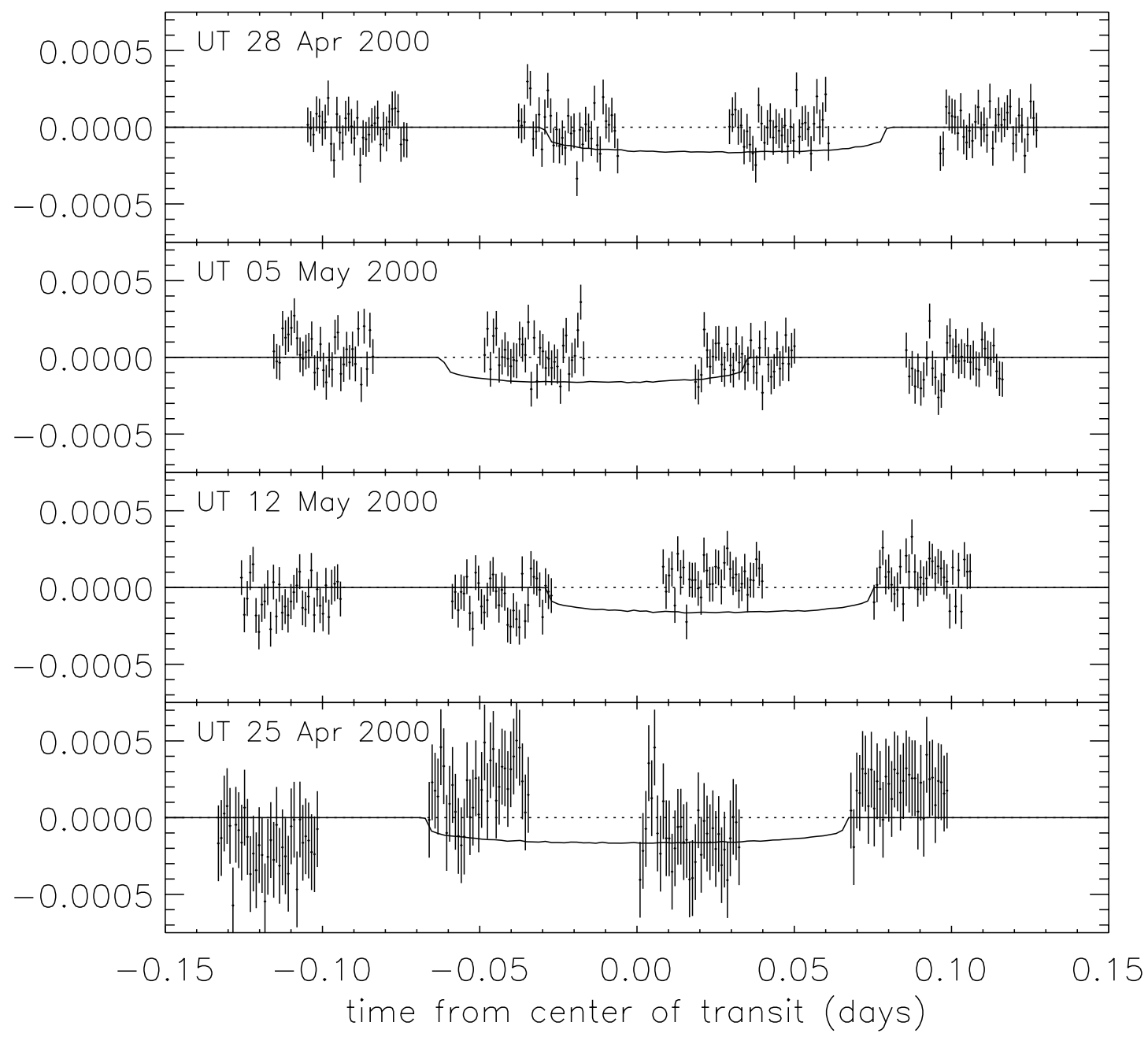

FIG. 8.- Residuals around the fit of a five-parameter transit model (see text) to the observations, shown with error bars. The solid lines show the light curve one would expect given the presence of a satellite of $1.5 R_{\oplus}$, with an orbital period of 1.5 days. Note the relatively large errors for the first transit, which are due to the offset of the spectrum in the CCD subarray.

amount of time by which the center of the satellite transit would lead or trail the center of the planetary transit. We then subtracted these corrections from the times of the observations (so as to phase the data to any potential satellite transit), yielding the new times $t_{p, \phi}(i)$. Neglecting small changes in the duration of the satellite transit due to satellite orbital motion during the transit times, we assumed the duration of the satellite transit, $d_{\text {sat }}$, to be the same as that of the planetary transit, 184.25 minutes. We then computed the correlation of these data with a boxcar function defined by

$$
B_{p, \phi}(i)= \begin{cases}1 & \text { if }\left|t_{p, \phi}(i)\right| \leq d_{\text {sat }} / 2 \\ -1 & \text { if }\left|t_{p, \phi}(i)\right|>d_{\text {sat }} / 2\end{cases}
$$

The correlation was given by

$$
C_{p, \phi}=\frac{2 \sum_{\mathrm{i}=1}^{N} B_{p, \phi}(i) R(i)}{N}
$$

where the leading factor of 2 accounts for the fact that any such satellite transit will have a mean of zero, since we had already subtracted the best-fit planetary transit. In the case of noiseless data, the value of $C_{p, \phi}$ at the correct $\{p, \phi\}$ is the depth of the transit: For a satellite of radius $R_{\text {sat }}$ passing in front of HD 209458, we would have $C_{p, \phi} \simeq 6.4$ $\times 10^{-5}\left(R_{\text {sat }} / R_{\oplus}\right)^{2}$.

To derive detection thresholds, we replaced all the residuals with numbers drawn from a Gaussian distribution with $\sigma=1.14 \times 10^{-4}$ and evaluated $C_{p, \phi}$. We repeated this many times, and evaluated the maximum value of $C_{p, \phi}$ for each fake data set. These maximum values were Gaussiandistributed, with the upper $3 \sigma$ point of the distribution lying at $C=5.2 \times 10^{-5}$. Thus, assuming the noise to be purely Gaussian, we exclude with $99.7 \%$ confidence the presence of satellites larger than the corresponding radius, namely, $R_{\text {sat }}=0.9 R_{\oplus}$.

The above conclusion is correct only if the errors are indeed Gaussian. However, we can see from the residuals that there may exist orbit-to-orbit drifts. To account for these as well, we performed a different test. For each orbit, we calculated the average of the residuals and found the maximum average offset to be $6.9 \times 10^{-5}$. As before, we replaced all the residuals with numbers drawn from a Gaussian distribution with $\sigma=1.14 \times 10^{-4}$. We then added to each orbit of data an additional offset drawn from a uniform distribution between $\pm 6.9 \times 10^{-5}$. We then evaluated $C_{p, \phi}$, and repeated this procedure many times. Following the same procedure as before, we found a $3 \sigma$ 
limit of $C=9.0 \times 10^{-5}$, corresponding to $R_{\text {sat }}=1.2 R_{\oplus}$. We believe that this noise model better describes our data than does pure Gaussian noise, so we prefer this estimate of the detection threshold. The smaller threshold remains of interest, however, as an indication of the size of detectable companions, should the remaining systematic errors of measurement be eliminated.

For our actual residuals, the maximum of $C_{p, \phi}$ is $4.88 \times 10^{-5}$. This value is significantly below the detection threshold described in the last paragraph and consistent with the result of pure noise. We conclude that we have no evidence for a satellite orbiting the planet of HD 209458, and can exclude (at the $3 \sigma$ level) satellites larger in radius than $1.2 R_{\oplus}$. These results show that if we obtained the same precision on a star with $R_{*}=1.0 R_{\odot}$ (recall that for HD 209458, $R_{*}=1.15 R_{\odot}$ ), we would be able to make a $3 \sigma$ detection of a $1.0 R_{\oplus}$-sized planet in transit across its star. In the absence of orbit-to-orbit drifts, the limit would be $0.78 R_{\oplus}$.

Satellites might also be detected by the periodic displacement of the planet in its orbit due to the gravitational attraction of the satellite. The magnitude $\delta x$ of this displacement could be as much as $\delta x=a_{\text {sat }} M_{\text {sat }} / M_{p}$, where $a_{\text {sat }}$ is the satellite's orbital radius and $M_{\text {sat }}$ is its mass. The visible effect would be to wobble the planet ahead of or behind its mean orbital phase, assuming the satellite orbit to be approximately coplanar with that of the planet. Transits would therefore occur early or late relative to the ephemeris, depending upon the phase of the satellite in its orbit. For an $1 M_{\oplus}$ satellite orbiting HD 209458b at a distance of one Hill sphere radius, the maximum temporal excursion is $13 \mathrm{~s}$. This time is comparable to the formal $3 \sigma$ error on the estimate of the central time of a single transit. Relative to the time predicted from a best-fit estimate (based on these HST data only) of the period and initial epoch, the observed timing displacements (seconds) for the four transits are $\{-20.0 \pm 10.0,0.3 \pm 4.4,-3.3 \pm 6.2,10.0 \pm 4.6\}$. Figure 9 shows these observed displacements. Also shown is the $\chi^{2}$ statistic for each transit, as a function of displacement from the observed transit times. From these curves, it is evident that the differences between the observed transit times and the predicted ones are at most about $2 \sigma$. We

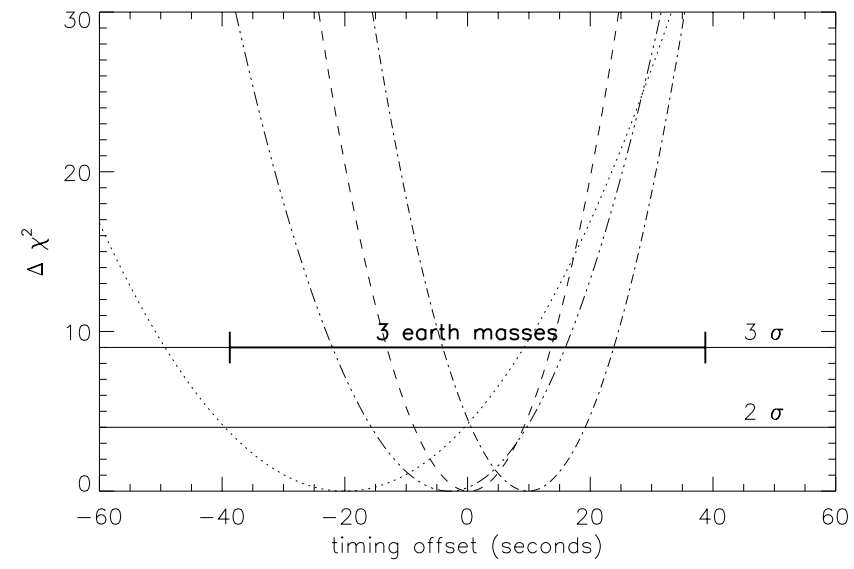

Fig. 9. - The $\chi^{2}$ curves for fits to the central time of transit for each of the four transits (dotted line $=25$ April, dashed line $=28 / 29$ April, tripledot dashed line $=5 / 6$ May, dot-dashed line $=12 / 13$ May). The horizontal bar shows the range of timings resulting from a $3 M_{\oplus}$ satellite at a distance of 1 Hill sphere radius. doubt these discrepancies arise from satellites of the planet. Rather, we suspect that small systematic errors in the observations (for example, small linear trends in detector sensitivity that run from beginning to end of a 5-orbit transit observation) may be responsible. The residuals shown in Figure 8 support this conclusion, with the residuals for the transit on May 12 being predominantly negative before the transit and positive after it; residuals due to a planetary satellite should affect the in-transit data (orbits 3 and 4), but not the out-of-transit data (orbits 2 and 5). Taking the observed transit time variations to be an upper limit on the displacement caused by an unseen satellite, and assuming its orbital radius to be that of the Hill sphere, we can exclude with $3 \sigma$ confidence the presence of satellites of more than $3 M_{\oplus}$ ).

\section{DISCUSSION}

The planetary radius $1.347 R_{\text {Jup }}$ inferred from the $H S T$ light curve is consistent with a previously reported value of $1.40 R_{\text {Jup }}$ (Mazeh et al. 2000; Henry et al. 2000), and somewhat smaller than the value of $1.55 R_{\mathrm{Jup}}$ derived from multicolor data by Jha et al. (2000). The new estimate is more likely to be correct, because the radius of the parent star is determined as part of the fitting process, rather than being assumed. Indeed, the works just cited identified uncertainty in the stellar radius as an important contributor to the uncertainty of the final result. An assumption about the stellar mass is required in any case, but our errors include a $10 \%$ uncertainty in the stellar mass. Furthermore, the derived value for the planetary radius is only weakly dependent upon the value of the stellar mass. A radius of 1.35 $R_{\text {Jup }}$ is consistent with the irradiated model described by Burrows et al. (2000); it falls between the models with high $(0.5)$ and low (0.0) Bond albedo, and the error of $0.05 R_{\mathrm{Jup}}$ is, in principle, small enough to distinguish between these two albedos. Implied values of the mean density $\rho$, surface gravity $g$, and escape velocity $v_{e}$ all increase relative to earlier estimates, but not by large amounts. We find $\rho=0.35 \mathrm{~g} \mathrm{~cm}^{-3}, g=943 \mathrm{~cm} \mathrm{~s}^{-2}$, and $v_{e}=43 \mathrm{~km} \mathrm{~s}^{-1}$. These changes are all such as to increase the estimated stability of the planet against disruption by tidal forces, thermal evaporation, or mass stripping by the stellar wind.

Although it was possible a priori for the system to have observably large satellites or rings, the absence of these features is not surprising. Only large satellites (bigger than the Earth) could have been detected by these observations, and for a satellite to survive so close to the star, it would have to be made of refractory materials. The solar system contains no bodies that meet both of these requirements (Earth and Venus come the closest), so perhaps it is reasonable to guess that HD 209458b likewise is not home to such an object. Similar comments apply to a ring system, which would have to be large and opaque in order be detectable in our observations. In the harsh radiation environment $0.05 \mathrm{AU}$ from the central star, the processes that destroy rings of fine particles would be accelerated, and long lifetimes may not be expected. One must remember, however, that the present observations could not have detected the Galilean satellites of Jupiter, and (because of their small optical depth) the rings of Saturn would be only marginally visible. Our observations constrain the presence of such objects only to the extent that large and obvious companions are excluded.

In addition to their direct scientific interest, these observations provide the best example to date of the capabilities 
of extremely precise photometry from space. This suggest a number of potentially rewarding future observations:

1. With the achieved precision, it may be possible to detect the reflected light from a close-in giant planet (Seager \& Sasselov 1998; Sudarsky, Burrows, \& Pinto 2000; Marley et al. 1999). To date, such studies (Charbonneau et al. 1999; Collier Cameron et al. 1999) have produced only upper limits and have required restrictive assumptions about the spectrum of the reflected light. The amplitude of this effect in the HD 209458 system would be $\epsilon_{\lambda}=$ $p_{\lambda}\left(R_{p} / a\right)^{2}=p_{\lambda} \times 2.0 \times 10^{-4}$, where $p_{\lambda}$ is the wavelengthdependent geometric albedo. The transiting configuration of HD 209458b would make it the ideal target, since at times of secondary eclipse (i.e., just as the planet passes behind the star), the planet would pass within 26 minutes from being nearly fully illuminated to not visible. For such observations where there is no particular interest in moderate-resolution spectroscopy (as was the case for this program), it would be desirable to switch to the lowresolution gratings, and thus measure the albedo across the wavelength range where the central star outputs the majority of its energy. One could thus evaluate the net energy deposition into the planet, a key quantity in understanding its evolution (Burrows et al. 2000).

2. The perturbation caused by additional planets in the HD 209458 system would change the observed times of transit. A $1 M_{\text {Jup }}$ planet at $10 \mathrm{AU}$ would cause the central star to move $\pm 0.01 \mathrm{AU}$, and thus the transits would be observed as much as $5 \mathrm{~s}$ earlier or later. This effect could be used to infer the presence of such additional companions, although many years would be required to observe the effect, owing to the long orbital periods at these large semimajor axes.

3. Although the STIS instrument was not designed with high SNR photometry in mind, the achieved precision of the photometric time series confirms that it is feasible to detect the transits of Earth-sized planets across the disks of Sun-like stars. Moreover, reaching this precision does not depend upon the HST's large aperture; the limited bandwidth used in this experiment and (to a lesser degree) the transmission losses in the spectrograph combined to make the photometry far less efficient than it could be. For example, with a bandwidth spanning 400-1000 $\mathrm{nm}$ and a system efficiency of $50 \%$, the photon count rates achieved here could be reached with a telescope of only $25 \mathrm{~cm}$ aperture. Placed in a suitable orbit, so that full uninterrupted transits of HD 209458b could be observed, such a telescope could detect a satellite of the mass or radius of Ganymede after observing about 100 transits, which is to say, within about 1 yr. The first generation of orbiting telescopes designed for such purposes are now under development (MOST [Matthews et al. 2000]; COROT [Michel et al. 2000]; MONS [Kjeldsen, Bedding, \& ChristensenDalsgaard 2000]), and larger scale projects are planned or proposed, such as the Kepler Mission (Koch et al. 1998) and Eddington. ${ }^{7}$

With the new photometric and spectroscopic data sets that will soon become available, we can look forward to an exciting decade unraveling the structure and history of the close-in extrasolar planets.

We are grateful to the HST STIS and operations teams, especially Helen Hart, Gerard Kriss, and Jeff Valenti for their prompt and insightful help in resolving the database error experienced during the first transit. Support for proposal HST-GO-08789.01-A was provided by NASA through a grant from the Space Telescope Science Institute, which is operated by the Association of Universities for Research in Astronomy, Inc., under NASA contract NAS 5-26555. Further support for this work was provided through NASA grants NAG 5-7073 and NAG 5-7499. D. C. is supported in part by a Newkirk Fellowship of the High Altitude Observatory.

${ }^{7}$ See http://astro.esa.int/SA-general/Projects/Eddington.

\section{REFERENCES}

Brown, T. M. 2001, astro-ph/0101307

Brown, T. M., Charbonneau, D., \& Gilliland, R. L. 2001, AAS Meeting 196,0203

Burrows, A., Guillot, T., Hubbard, W. B., Marley, M. S., Saumon, D., Lunine, J. I., \& Sudarsky, D. 2000, ApJ, 534, L97

Castellano, T., Jenkins, J., Trilling, D. E., Doyle, L., \& Koch, D. 2000, ApJ, 532 , L51

Charbonneau, D., Brown, T. M., Latham, D. W., \& Mayor, M. 2000, ApJ, 529, L45

Charbonneau, D., Noyes, R. W., Korzennik, S., Nisenson, P., Jha, S., Vogt, S. S., \& Kibrick, R. I. 1999, ApJ, 522, L145

Claret, A., \& Gimenez, A. 1990, A\&A, 230, 412

Collier Cameron, A., Horne, K., Penny, A., \& James, D. 1999, Nature, 402, 751

Cox, A. N. 1999, Allen's Astrophysical Quantities (New York: Springer), 355

Doyle, L. R., et al. 2000, ApJ, 535, 338

Gilliland, R. L. 1999, STIS Instrument Science Report 99-05 (Baltimore: STSci)

Gilliland, R. L., Goudfrooij, P., \& Kimble, R. A. 1999, PASP, 111, 1009

Gilliland, R. L., et al. 2000, ApJ, 545, L47

Guillot, T. 1999, Science, 286, 72

Guillot, T., Burrows, A., Hubbard, W. B., Lunine, J. I., \& Saumon, D. 1996, ApJ, 459, L35

Henry, G. W., Marcy, G. W., Butler, R. P., \& Vogt, S. S. 2000, ApJ, 529, L41

Høg, E., et al. 2000, A\&A, 355, L27
Jha, S., Charbonneau, D., Garnavich, P. M., Sullivan, D. J., Sullivan, T., Brown, T. M., \& Tonry, J. L. 2000, ApJ, 540, L45

Kjeldsen, H., Bedding, T. R., \& Christensen-Dalsgaard, J. 2000, in ASP Conf. Ser. 203, The Impact of Large-Scale Surveys on Pulsating Star Research, ed. L. Szabados \& D. Kurtz (San Francisco: ASP), 73

Koch, D. G., Borucki, W., Webster, L., Dunham, E., Jenkins, J., Marriott, J., \& Reitsema, H. J. 1998, Proc. SPIE, 3356, 599

Marley, M. S., Gelino, C., Stephens, D., Lunine, J. I., \& Freedman, R. 1999, ApJ, 513, 879

Matthews, J. M., et al. 2000, in ASP Conf. Ser. 203, The Impact of LargeScale Surveys on Pulsating Star Research, ed. L. Szabados \& D. Kurtz (San Francisco: ASP), 74

Mazeh, T., et al. 2000, ApJ, 532, L55

Michel, E., et al. 2000, in ASP Conf. Ser. 203, The Impact of Large-Scale Surveys on Pulsating Star Research, ed. L. Szabados \& D. Kurtz (San Francisco: ASP), 69

Perryman, M. A. C., et al. 1997, A\&A, 323, L49

Robichon, N., \& Arenou, F. 2000, A\&A, 355, 295

Rosenblatt, F. 1971, Icarus, 14, 71

Sackett, P. D. 1999, in Planets Outside the Solar System: Theory and Observations, ed. J.-M. Mariotti \& D. M. Alloin (NATO/ASI Ser C, 532; Dordrecht: Kluwer), 189

Sartoretti, P., \& Schneider, J. 1999, A\&AS, 134, 553

Schneider, J. 1999, CR Acad. Scie. Ser. II, 327, 621

Seager, S., \& Sasselov, D. D. 1998, ApJ, 502, L157

2000, ApJ, 537, 916

Sudarsky, D., Burrows, A., \& Pinto, P. 2000, ApJ, 538, 885 